\title{
THE COUPLING CORRECTION SYSTEM AT RHIC: RESULTS FOR THE RUN 2000 AND PLANS FOR 2001*
}

\author{
F. Pilat, W. Fischer, S. Peggs, V. Ptitsyn, S. Tepikian, BNL, New York, USA
}

\section{Abstract}

The RHIC coupling correction system has been commissioned during the Year 2000 run, which marked the successful first year of operation of the machine. The RHIC coupling correction system is described with particular emphasis on its flexibility, which allows using both global and local coupling compensation techniques.

Coupling measurements and correction data are presented for the RHIC Blue and Yellow rings, together with the procedure used to reduce the minimum tune separation to 0.001 , the typical resolution for tune measurements during run 2000 . We further demonstrate how local coupling compensation in the interaction regions substantially reduces the strength of the skew quadrupole families used for global coupling compensation.

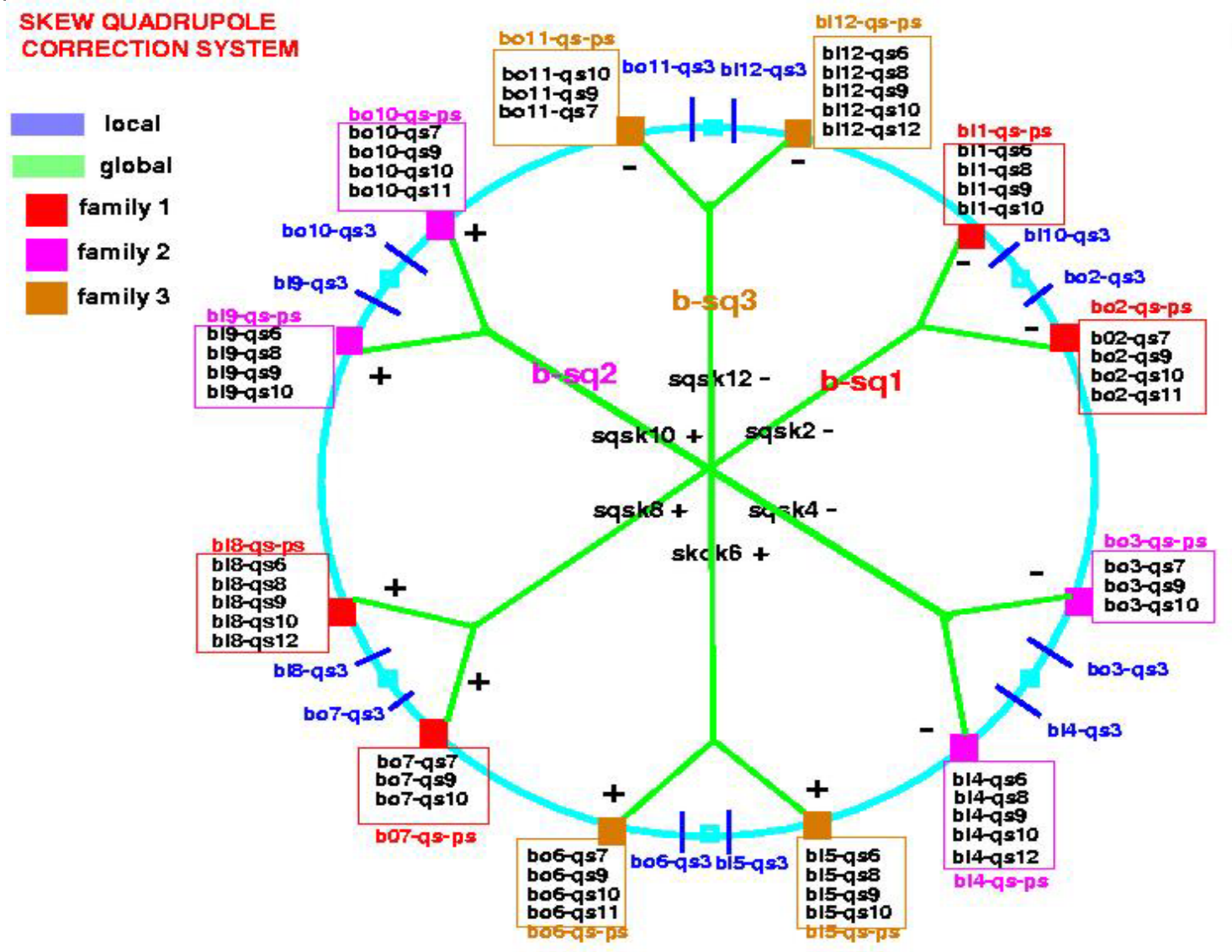

Figure 1: Schematic view of the skew quadrupole correction system in the RHIC Blue ring.

\section{THE CORRECTION SYSTEM}

The RHIC coupling correction system is schematically described in Figure 1 for the Blue ring, the system for the Yellow ring being an exact copy of this. A total of 48 skew quadrupoles per ring are wired up in 3 families to allow global coupling corrections, and 12 independently powered skew quadrupoles are located in the Interaction Region (IR) triplets to allow for local correction of coupling sources in the IR's.
Every skew family includes 16 skew quadrupoles, located in regions adjacent to the IR's; four power supplies per family power the skew quadrupoles in strings of three to five magnets. This powering scheme optimizes the logistics of power supply positioning in the ring service building and adds degrees of freedom to the system. The 4 power supplies in the family are set to the same current in software. By powering with opposite currents skew quadrupoles in the same family but opposite positions in the ring (see Figure 1), their effect adds up, given the 
lattice phases. The skew families are used to minimize the tune separation at the coupling resonance: for this, a minimum of 2 orthogonal knobs is needed. The RHIC system has 3 families by design, to follow the 6-fold symmetry of the lattice and they are about 120 degree apart. Family 2 and 3 can be combined to provide a vector orthogonal to Family 1 if desired. The extra degree of freedom however can be used to minimize the required strength.

The skew quadrupoles for local IR correction are nested in the multi-layer corrector package positioned between the Q2 and Q3 triplet quadrupoles and are each independently power by bipolar 50 A supplies.

\section{MODELING OF PERFORMANCE}

The system can be used in several ways to correct coupling in the machine. Prior to RHIC commissioning and operations, coupling sources were analyzed, different coupling corrections techniques simulated [1]. The calibration and performance of the system was analyzed in term of $\Delta$ Qmin (minimum tune separation), or the width of the coupling resonance.

The main coupling sources in RHIC are: skew (a1) errors in arc dipoles, roll alignment errors in quadrupoles (and particularly IR triplets), helical devices (spin Siberian snakes and rotators), axial fields (Phenix experiment) [2] and vertical offsets in sextupoles. Table 1 compares the effect of coupling sources at collision with the strength of the correction system and measurements in term of $\Delta \mathrm{Qmin}$.

\begin{tabular}{|l|l|}
\hline Configuration & $\Delta$ Qmin \\
\hline Systematic a1 $=4$ units in all dipoles & 0.051 \\
\hline Random roll $0.5 \mathrm{mrad}$ in all quadrupoles & 0.058 \\
\hline Random roll $0.5 \mathrm{mrad}$ in IR triplets & 0.043 \\
\hline Random roll $0.5 \mathrm{mrad}$ (worst seed) & 0.131 \\
\hline $\begin{array}{l}\text { Maximum corrector strength at collision } \\
(3 \text { families at } 50 \mathrm{~A}-\mathrm{kL}=0.001785 \mathrm{~m}-1)\end{array}$ & 0.197 \\
\hline
\end{tabular}

Table 1. Comparison of coupling sources effects and corrector strength

Analysis of the above table allows us to conclude that at collision the effect of misalignment mainly arises from the triplets, and that we have enough corrector strength in the system. Note that the integer split in the tunes (design tunes 28.19 29.18) makes the coupling correction much easier. (A -2.5 systematic a1 error in arc dipoles generates a $\Delta \mathrm{Qmin}=0.2$ without the integer split and $\Delta \mathrm{Qmin}=0.02$ with the split tunes).

The strategy for coupling correction is the following:

1. Local correction of IR effects (from triplets and axial fields) with the local IR skew quadrupoles, locally minimizing the off-plane rms orbit. [3]

2. Global correction of residual coupling sources (arc magnets, helical devices, etc.) with the skew families, operationally finding and minimizing $\Delta \mathrm{Qmin}$.
3. Measurement of local coupling using 1000 turns beam position monitor (BPM) data and local compensation. [4]

\begin{tabular}{|l|l|l|l|}
\hline $\begin{array}{l}\text { Skew } \\
\text { quadrupoles }\end{array}$ & $\begin{array}{l}\text { Strength } \\
{[\mathrm{m}-1]}\end{array}$ & $\begin{array}{l}\Delta \text { Qmin } \\
\text { calculation }\end{array}$ & $\begin{array}{l}\Delta \text { Qmin } \\
\text { simulation }\end{array}$ \\
\hline Fam1 (b-sq1) & 0.0005 & 0.032 & 0.028 \\
\hline Fam2 (b-sq2) & 0.0005 & 0.032 & 0.029 \\
\hline Fam3 (b-sq3) & 0.0005 & 0.032 & 0.029 \\
\hline Skew at IP2 & 0.0005 & 0.025 & 0.024 \\
\hline
\end{tabular}

Table 2. Calibration of skew strengths in terms of $\Delta \mathrm{Qmin}$.

\section{BEAM MEASUREMENTS AND MACHINE COUPLING CORRECTION}

\subsection{RHIC Run 2000}

The Run 2000 marked the successful commissioning and first year of operation. The focus of coupling correction has been tune control at injection and during the ramps. We commissioned the global coupling correction system and gathered beam data and experience to set up the local skew correction system at the IR's.

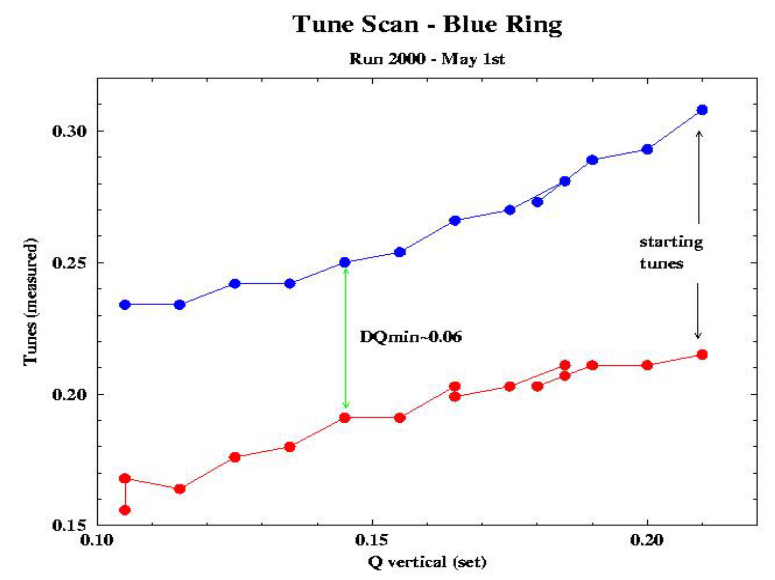

Figure 2. First tune scan in RHIC to measure $\Delta$ Qmin in the Blue Ring.

\begin{tabular}{|l|l|}
\hline Machine configuration & $\Delta$ Qmin \\
\hline $\begin{array}{l}\text { Measured uncorrected } \Delta \text { Qmin (injection) } \\
1999 \beta^{*=}=2.5 \mathrm{~m} \text { symmetric lattice (blue) }\end{array}$ & 0.03 \\
\hline $\begin{array}{l}\text { Measured uncorrected } \Delta \text { Qmin (injection) } \\
2000 \beta^{*}=3 \text { to } 8 \mathrm{~m} \text { asymmetric lattice (blue) }\end{array}$ & $0.06 . /$ \\
\hline $\begin{array}{l}\text { Measured } \Delta \text { Qmin (injection) } \\
2001 \beta^{*}=10 \mathrm{~m} \text { symmetric lattice (blue) }\end{array}$ & 0.009 \\
\hline
\end{tabular}

Table 3. Comparison of uncorrected minimum tune separation in the RHIC blue ring during successive runs.

During Run 2000 the measured tune separation was measured to be 0.06, twice the value measured in 1999 because we used an asymmetric lattice with 2 IR's tuned to $\beta^{*}=3 \mathrm{~m}$ and 4 IR's at $8 \mathrm{~m}$. (see Table 3 ). 
The Blue ring was corrected to $\Delta \mathrm{Qmin} \sim 0.002$, that is to the resolution of tune measurements in 2000 with the following settings for the families:

$\mathrm{k}(\mathrm{b}-\mathrm{sq} 1)=0.0008, \mathrm{k}(\mathrm{b}-\mathrm{sq} 2)=0.0006$ and $\mathrm{k}(\mathrm{b}-\mathrm{sq} 3)=-0.0003$. The correction of the Yellow ring was likewise done but in order to achieve a $\Delta \mathrm{Qmin} \sim 0.007$ we had to push family 1 to the large strength of 0.0016 . Analysis of local coupling data in the IR's with orbit bumps [3] allowed us to identify a large coupling source in one of the IR8 triplets in the yellow ring, likely an alignment error in one of the triplet quadrupoles. After the local compensation at IP8, the global families were used to correct the residual coupling in the ring: the strength of family 1 , in phase with the coupling source at IP8, was reduced by a factor $\sim 3$ to 0.00055 , and $\Delta$ Qmin was minimized to 0.001 as can be seen by the scan in Figure 3 .

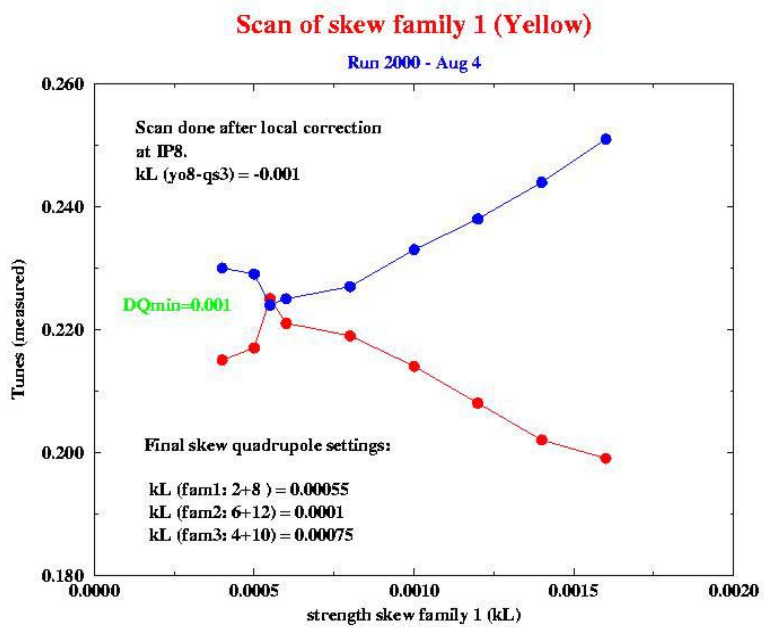

Figure 3. Scan of skew quadrupole family 1 in the Yellow ring.

\subsection{RHIC Run 2001}

The RHIC run 2001 started in May 2001 and will continue till early 2002. With the full complement of power supplies now installed in the machine, the lattice at injection is the design lattice now, symmetric with $\beta^{*}=10 \mathrm{~m}$ at all IR's, with the final configuration at collision at $\beta^{*}=2 \mathrm{~m}$ at all IR's.

During the RHIC 2000 run systematic data about the effect of the triplets were taken: orbit rms and tune shifts were recorded and later analyzed as a function of bump amplitude at the triplets location. This analysis allowed us to predict settings for the local skew quadrupole correctors in the IR's. [3]. When we started with the Blue ring, we first set up the local skew quadrupole correctors in the IR's operationally and the resulting experimental setting were in very good agreement with the predictions. The minimum tune separation of the bare machine with the symmetric injection lattice was measured to 0.009 .
The family strengths to compensate for the residual coupling are :

$\mathrm{k}(\mathrm{b}-\mathrm{sq} 1)=0.0002, \mathrm{k}(\mathrm{b}-\mathrm{sq} 2)=0.0, \mathrm{k}(\mathrm{b}-\mathrm{sq} 3)=-0.0002$, weaker than during run 2000. An starting tune scan for the Blue ring is shown in Figure 4. The final correction achieved is to $\Delta \mathrm{Qmin}=0.0005$, the tune meter resolution for 2001 .

The plans for the rest of the run are first to correct the Yellow ring, and then to speed up the global correction. That can be accomplished by measuring tunes with the phase lock loop (PLL) while the tunes are continuously varied to determine the $\Delta \mathrm{Qmin}$, when the PLL system will become operational.

Another improvement that is planned is the measure and possible correction of local coupling effects by the analysis of 1000 turn data now available at every beam position monitor in the machine, by analyzing the in plane and out of plane response to kicks. We will initially use the tune meter kicker and later on an $\mathrm{AC}$ dipole when it will become available.

\section{CONCLUSIONS}

The RHIC coupling correction system that integrates local IR correction and global minimum tune compensation has been successfully commissioned during the RHIC run 2000 and improved during the run 2001. Further developments are planned for the rest of the 2001 run.

[1] F.Pilat, RAP Note 56

\section{REFERENCES}

[2] T.Satogata, RAP Note 19

[3]V.Ptitsyn, J.Cardona, J-P. Koutchok, F.Pilat, "Measurement and correction of linear effects in the RHIC Interaction regions", These Proceedings.

Decoupling scan - the first family was set to +0.00022

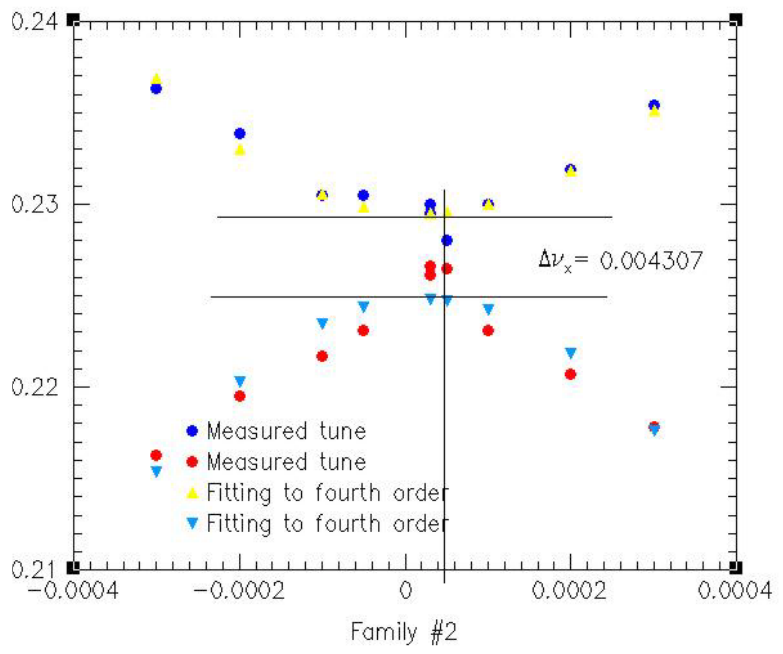

Figure 4: Coupling correction for the Blue ring in 2001. 